\title{
Neuropsychological functioning in first-episode schizophrenia
}

Eugenia Kravariti, Kevin Morgan, Paul Fearon, Jolanta W. Zanelli, Julia M. Lappin, Paola Dazzan, Craig Morgan, Gillian A. Doody, Glynn Harrison, Peter B. Jones, Robin M. Murray and Abraham Reichenberg

\section{Background}

Identifying neurocognitive subtypes in schizophrenia may help establish neurobiologically meaningful subtypes of the disorder, but is frequently confounded by differences in intellectual function between individuals with schizophrenia and controls.

\begin{abstract}
Aims
To examine neuropsychological performance in individuals with epidemiologically based, first-onset schizophrenia and
\end{abstract} intellectually matched controls.

\section{Method}

Using standard IQ and reading tests, we examined the proportions of 101 people with epidemiologically derived, first-onset schizophrenia/schizoaffective disorder and 317 community controls, falling into three a priori defined intellectual categories: 'stable good', 'deteriorated poor' and 'stable poor'. Neuropsychological function was compared between intellectually matched participants with schizophrenia and control subgroups.

\section{Results}

Multiple deficits in executive function, processing speed and verbal memory, but not visual/spatial perception/memory, were detected in all participant groups with schizophrenia compared with controls. The average effect size across the affected domains ranged from small to medium to large in the stable good, deteriorated poor and stable poor subgroups of participants with schizophrenia, respectively.

\section{Conclusions}

Compared with intellectually matched controls, people with epidemiologically derived, first-onset schizophrenia/ schizoaffective disorder show multiple deficits in executive function, processing speed and verbal memory.

\section{Declaration of interest}

None.
Intellectual impairment is a reliable correlate of schizophrenia, but it also determines, and frequently confounds, neuropsychological function in the disorder. ${ }^{1-7}$ Affected individuals with different IQ levels and trajectories are distinguishable in terms of neuropsychological performance, although abnormalities in certain cognitive processes, particularly executive functions and processing speed, may overlap across IQ categories, and coexist with intact intellect. ${ }^{3-6,8-10}$ Establishing neurocognitive commonalities across levels and trajectories of IQ is important for characterising abnormalities that are prototypical of schizophrenia, ${ }^{6,9,10}$ and identifying intellectual or neuropsychological subtypes may help establish neurobiologically meaningful disease subtypes. ${ }^{8,11,12}$ Earlier investigations of people with schizophrenia with different intellectual characteristics had several methodological limitations. Most studies used clinical rather than epidemiologically based samples, ${ }^{3,4,6,13}$ and included individuals with chronic schizophrenia with long-term exposure to antipsychotic medication. ${ }^{3,4,6}$ All studies defined subgroups on the basis of either current IQ ${ }^{6}$ or a combination of estimated premorbid IQ and degree of decline from premorbid levels, ${ }^{3-5}$ but not both criteria combined. Finally, no study compared subgroups of people with schizophrenia, defined using both of the above criteria, with intellectually matched controls. ${ }^{7}$ To address this gap in the literature, the present study explored neuropsychological function in intellectually different subgroups of individuals with first-episode schizophrenia/ schizoaffective disorder and controls with no history of psychosis, derived from an epidemiological sample. Intellectual categories were generated using similar methods to those employed in previous studies, but were more refined. In contrast to earlier studies, group formation was based on both current IQ and degree of discrepancy from estimated premorbid levels, and participants with schizophrenia and controls were matched on both of the latter two criteria (in the case of controls, 'premorbid' denotes 'prior'). We hypothesised that impairment in executive function and processing speed would be detected in all participant groups with schizophrenia compared with intellectually matched controls.

\section{Method}

\section{The AESOP study}

The data were derived from the baseline ÆSOP (Aetiology and Ethnicity in Schizophrenia and Other Psychoses) study, a major population-based, case-control study of first-episode psychosis. The study identified all individuals aged 16-65 years with a first episode of psychosis (F10-F29 and F30-F33 in ICD-10) $)^{14}$ who presented to specialist mental health services in tightly defined catchment areas in south-east London, Nottingham and Bristol between September 1997 and August 1999. Local ethical committees at all three centres approved the study. Exclusion criteria were previous contact with health services for psychosis, organic causes of psychotic symptoms, transient psychosis due to acute intoxication (as defined by ICD-10) and IQ less than 50. The study further included a random sample of controls with no past or present psychotic disorder. Community controls were mainly recruited using a sampling method that matched them with participants with schizophrenia by area of residence. A detailed overview of the ÆSOP study has been published elsewhere. ${ }^{15}$

\section{Participants}

One hundred and one people with schizophrenia or schizoaffective disorder (F20 or F25 in ICD-10; $n=85$ and $n=16$, 
respectively) and 317 community controls were investigated to establish whether they met criteria for allocation to one of six a priori defined intellectual categories (see Generation of IQ categories below). In addition to satisfying the general ÆSOP inclusion criteria, all participants were selected if they: had IQ measurements on the National Adult Reading Test - Revised $(\text { NART-R })^{16}$ and a short form of the Wechsler Adult Intelligence Scale - Revised (WAIS-R), ${ }^{17}$ as well as at least one other score on the ÆSOP neuropsychological battery; and were native speakers of English or had migrated to the UK by the age of 11 . The latter strategy ensured that participants with a good command of English as a non-native language had completed at least their secondary education in the UK, thus reducing linguistic or cultural effects on the performance of a multi-ethnic sample. After providing the participants with a complete description of the study written informed consent was obtained.

There were no differences in age, gender or years of education between individuals with schizophrenia/schizoaffective disorder who did not meet the language/migration criteria or had no neuropsychological/IQ data $(n=41)$ and those participating (all $P>0.1$ ), although the former showed a significantly higher Black African to White ratio (Fisher's exact $P<0.001$ ).

\section{Diagnostic procedures}

Clinical data were collected using the Schedules for Clinical Assessment in Neuropsychiatry (SCAN). ${ }^{18}$ The Present State Examination - Version 10 (incorporated in the SCAN) was used to elicit symptom-related data at presentation. Where a patient interview was not possible, case notes were used to complete the SCAN Item Group Checklist. Patients' ICD-10 diagnoses were determined using the SCAN data on the basis of consensus meetings involving a principal investigator and other members of the research team. Interrater reliability was estimated by asking each principal investigator to independently formulate a diagnosis for 20 individuals based on the same SCAN information (kappa values ranged from 0.63 to $0.75, P<0.001$ ).

Controls were screened for psychosis using the Psychosis Screening Questionnaire. ${ }^{19}$ Those with a positive rating on this instrument were further assessed using the SCAN.

\section{Sociodemographic characteristics}

Data on age, gender, ethnicity and education were collected by interviews with the participants using the Medical Research Council Sociodemographic Schedule. ${ }^{20}$ Information gaps were filled using additional data sources, including case notes and other informants. In cases of uncertain ethnicity, a consensus rating was made by members of the research team having long-standing expertise in the study of ethnicity and mental health.

\section{Generation of IQ categories}

Premorbid and current IQs were derived, respectively, using the NART- $\mathrm{R}^{16}$ and a short form of the WAIS-R, ${ }^{17}$ comprising Vocabulary, Comprehension, Digit symbol and Block design.

Following the criticism by Kremen et $a l^{7}$ we used an adaptation of the Weickert et $a l^{3}$ classification: all participants were characterised as intellectually 'good' or 'poor' based on current IQ (in line with Wechsler's classification ${ }^{17}$ a score of 90 , the cut-off for 'average' IQ, was chosen as the cut-off for 'good' functioning), and as 'improved', 'stable' or 'deteriorated' based on degree of discrepancy between NART-R IQ and WAIS-R IQ. A NART-R minus WAIS-R discrepancy of 10 IQ points was used as the cut-off for a meaningful change (gain or decline) from estimated premorbid levels. This choice was based on previous studies, which showed that a 10-point discrepancy between current and premorbid IQ applied to less than $25 \%$ of the NART restandardisation sample ( $10 \%$ of healthy controls showed an IQ 'decline', whereas $13 \%$ showed an IQ 'gain', of this magnitude), ${ }^{16}$ represents the average deterioration between pre- and postschizophrenia, ${ }^{2,21}$ and differentiates effectively between neuropsychological subtypes of the disorder. ${ }^{3-5}$ In addition, in order to classify a participant as 'stable', both premorbid and current IQ had to fall on the same side of the cut-off for 'good' intellectual functioning. Thus, participants were allocated to one of six a priori defined IQ categories: 'improved good' (WAIS-R IQ $\geqslant 90$, gain $\geqslant 10$ points), 'improved poor' (WAIS-R IQ $<90$, gain $\geqslant 10$ points), 'stable good' (NART-R/WAIS-R IQs $\geqslant 90$, discrepancy $<10$ points), 'stable poor' (NART-R minus WAIS-R IQ $<90$, discrepancy $<10$ points), 'deteriorated good' (WAIS-R IQ $\geqslant 90$, decline $\geqslant 10$ points) and 'deteriorated poor' (WAIS-R IQ $<90$, decline $\geqslant 10$ points).

The number of individuals in the schizophrenia group falling within each of the above categories was $6(6 \%), 3(3 \%), 22(22 \%)$, $19(19 \%), 6(6 \%)$, and $37(37 \%)$, respectively, but 8 people $(8 \%)$ did not fall in any a priori defined category (e.g. discrepancy $<10$ points, but NART-R IQ and WAIS-R IQ did not fall on the same side of the cut-off for good functioning). The corresponding numbers of controls were $50(16 \%), 3$ (1\%), 137 (43\%), 14 (4\%), $44(14 \%)$ and $26(8 \%)$, but $43(14 \%)$ did not fall in any a priori defined category.

Participants with schizophrenia and controls differed significantly in their distribution in the 'improved', 'stable' and 'deteriorated' categories (Pearson $\chi^{2}=18.00, P=0.001$ ) (controls were over-represented in the 'improved' categories compared with participants with schizophrenia: $17 \%$ v. 9\%; individuals with schizophrenia were over-represented in the 'deteriorated' categories compared with controls: $43 \%$ v. $22 \%$ ). As a result of the small number of individuals with schizophrenia falling in the 'improved' $(n=9)$ and 'deteriorated good' $(n=6)$ subgroups, the latter IQ categories were not included in the analysis.

\section{Neuropsychological assessment}

Verbal learning, short-term verbal recall and delayed verbal recall were assessed using trials 1-5, 6 and 7, respectively, of the Rey Auditory Verbal Learning Test (RAVLT). ${ }^{22}$ Immediate visual memory was examined using Visual Reproduction I of the Wechsler Memory Scale - Revised (WMS-R). ${ }^{23}$ Working memory and executive function were evaluated using Trail Making - part $\mathrm{B},{ }^{24}$ Letter-number span, ${ }^{25}$ Raven's coloured progressive matrices $(\mathrm{CPM})-$ Set $\mathrm{B},{ }^{26}$ Category fluency (categories 'body parts', 'fruits' and 'animals'), and Letter fluency (letters F, A, S). Processing speed was measured using Trail Making - part $\mathrm{A}^{24}$ and the WAIS-R Digit symbol subtest. ${ }^{17}$ Raven's coloured progressive matrices - Set $\mathrm{A}^{26}$ and WAIS-R Block design ${ }^{17}$ were employed to assess visual and visuospatial perception and organisation, whereas WAIS-R Vocabulary ${ }^{17}$ and Comprehension ${ }^{17}$ were used to reflect verbal ability.

\section{Statistical analysis}

The statistical analysis was performed using STATA 8.0 for Windows. Differences in sociodemographic/IQ characteristics among the six participating groups (stable good schizophrenia group, stable good control group, deteriorated poor schizophrenia group, deteriorated poor control group, stable poor schizophrenia group, stable poor control group) were assessed using analysis of variance (ANOVA) models, Pearson chi-square and Fisher's 
exact tests. Differences in neuropsychological characteristics among the six groups were assessed using analysis of covariance (ANCOVA) models, controlling for age, gender, ethnicity and years of education (the latter variables showed significant/suggestive differences across groups; see Results). All AN(C)OVAs were performed using robust standard errors to safeguard against potential violations of the standard ANOVA assumptions. The AN $(C)$ OVAs that gave rise to significant results $(P<0.0027$ using a Bonferroni correction: $0.05 / 18$ neuropsychological and intellectual variables - including WAIS-R, NART-R and NART-R minus WAIS-R) were followed by planned post hoc contrasts between intellectually matched cases and controls ( $\alpha$ was set at 0.05 ). As significance tests are dependent on sample size (which ranged from small to large across groups), for each neuropsychological variable we further estimated the standardised difference (effect size) between the means of intellectually matched participants with schizophrenia and controls using Cohen's $d$ $\left(d=\mathrm{M}_{\text {cases }}-\mathrm{M}_{\text {controls }} / \sigma_{\text {controls }}\right)$. Cohen ${ }^{27}$ classified effect sizes ranging from 0.2 to 0.49 as small, from 0.5 to 0.79 as medium, and from 0.8 and above as large. As a deficit of half a standard deviation (equivalent to an effect size of 0.5 ) compared with established norms or healthy controls is regarded as the threshold of clinical or practical importance, we used this convention in addition to (or regardless of) statistical significance to characterise neurocognitive variables with a likely deficit in the people with schizophrenia. Negative effect sizes indicated better performance in the people with schizophrenia compared with their control counterparts.

\section{Results}

\section{Sociodemographic and intellectual characteristics}

Table 1 shows the demographic and intellectual characteristics of the six participating groups, which differed significantly in age, years of education, ethnicity, NART-R IQ, WAIS-R IQ, NART-R IQ minus WAIS-R IQ, and, at a trend level, in gender. The mean IQs of the schizophrenia $(n=78)$ and control $(n=177)$ samples were 95.7 (s.d. = 14.1) and 106.3 (s.d.=12.3), respectively, on the NART-R $\left(t_{253}=-6.08, P<0.0001\right)$, and $86.0($ s.d. $=15.1)$ and 102.9 (s.d. $=15.2)$, respectively, on the WAIS-R $\left(t_{253}=-8.24\right.$, $P<0.0001)$.

Within each IQ category (stable good, deteriorated poor, stable poor), there were no statistically significant differences/ trends in NART-R IQ, WAIS-R IQ or NART-R IQ minus WAIS-R IQ between participants with schizophrenia and controls (Table 1).

\section{Neuropsychological characteristics}

Table 2 shows the means and standard deviations of the six groups in the 15 neurocognitive variables (adjusted for age, gender, ethnicity and years of education), the number of participants in each statistical comparison, the results of the ANCOVAS and post hoc contrasts, and the standardised mean differences (Cohen's $d$ ) between intellectually matched individuals with schizophrenia

Table 1 Demographic and intellectual characteristics of individuals in the stable good, deteriorated poor and stable poor

schizophrenia and control groups

\begin{tabular}{|c|c|c|c|c|c|c|c|}
\hline \multirow[b]{2}{*}{ Variable } & \multirow[b]{2}{*}{$\begin{array}{l}\text { Group differences, } \\
\text { statistical test }\end{array}$} & \multicolumn{2}{|c|}{ Stable good } & \multicolumn{2}{|c|}{ Deteriorated poor } & \multicolumn{2}{|c|}{ Stable poor } \\
\hline & & $\begin{array}{l}\text { Schizophrenia } \\
\quad(n=22)\end{array}$ & $\begin{array}{l}\text { Control } \\
(n=137)\end{array}$ & $\begin{array}{l}\text { Schizophrenia } \\
\quad(n=37)\end{array}$ & $\begin{array}{l}\text { Control } \\
(n=26)\end{array}$ & $\begin{array}{l}\text { Schizophrenia } \\
\quad(n=19)\end{array}$ & $\begin{array}{l}\text { Control } \\
(n=14)\end{array}$ \\
\hline Age, years: ${ }^{a}$ mean (s.d.) & $F_{5,249}=10.94, P<0.0001$ & $27.0(7.7)$ & $38.2(13.4)$ & $27.2(8.9)$ & $33.9(8.1)$ & $26.7(11.8)$ & $34.1(13.0)$ \\
\hline Gender, $^{\mathrm{b}} n$ & $\chi^{2}=10.01, P<0.10$ & & & & & & \\
\hline Males & & 12 & 60 & 23 & 12 & 14 & 5 \\
\hline Females & & 10 & 77 & 14 & 14 & 5 & 9 \\
\hline Ethnicity, ${ }^{c} n$ & $\chi^{2}=53.58, P<0.0001$ & & & & & & \\
\hline White British & & 17 & 95 & 17 & 12 & 9 & 8 \\
\hline African-Caribbean & & 2 & 7 & 10 & 7 & 8 & 5 \\
\hline Black African & & 0 & 1 & 2 & 1 & 0 & 0 \\
\hline Asian & & 0 & 1 & 3 & 1 & 0 & 0 \\
\hline Other & & 3 & 33 & 5 & 5 & 2 & 1 \\
\hline Education, years: $^{\text {d }}$ mean (s.d.) & $F_{5,249}=13.14, P<0.0001$ & $13.7(2.4)$ & $13.6(2.4)$ & $12.3(2.1)$ & $12.5(2.3)$ & $11.3(1.0)$ & $11.9(1.7)$ \\
\hline Highest educational level attained, ${ }^{d} n$ & $\chi^{2}=75.29, P<0.001$ & & & & & & \\
\hline No qualifications & & 2 & 19 & 8 & 5 & 10 & 8 \\
\hline GCSE/CSE & & 3 & 20 & 13 & 9 & 5 & 2 \\
\hline 'O' Levels & & 2 & 9 & 5 & 4 & 2 & 1 \\
\hline 'A' Levels & & 5 & 34 & 2 & 0 & 1 & 0 \\
\hline Vocational/college & & 5 & 11 & 4 & 5 & 1 & 3 \\
\hline Teaching/HND/nursing & & 0 & 12 & 2 & 0 & 0 & 0 \\
\hline University/professional & & 5 & 32 & 3 & 3 & 0 & 0 \\
\hline NART-R IQ, ${ }^{e}$ mean (s.d.) & $F_{12,242}=61.10, P<0.0001$ & $107.2(8.5)$ & $109.6(10.3)$ & $98.1(10.3)$ & $101.3(8.9)$ & $77.5(5.7)$ & $83.3(5.5)$ \\
\hline WAIS-R IQ, ${ }^{\dagger}$ mean (s.d.) & $F_{12,242}=81.81, P<0.0001$ & $106.4(10.8)$ & $109.0(11.0)$ & $78.9(6.7)$ & $82.7(5.8)$ & $76.2(5.5)$ & $80.7(5.3)$ \\
\hline NART-R IQ - WAIS-R IQ, ${ }^{g}$ mean (s.d.) & $F_{12,242}=37.29, P<0.0001$ & $0.8(6.0)$ & $0.6(5.1)$ & $19.2(8.3)$ & $18.6(7.0)$ & $1.3(5.0)$ & $2.6(4.8)$ \\
\hline \multicolumn{8}{|c|}{$\begin{array}{l}\text { NART-R, National Adult Reading Test - Revised; WAIS-R, Wechsler Adult Intelligence Scale }- \text { Revised. } \\
\text { a. Planned post hoc contrasts between intellectually matched participants with schizophrenia and controls indicated that those with schizophrenia were statistically significantly } \\
\text { younger than controls in the stable good and deteriorated poor intellectual categories }(P<0.05) \text {. } \\
\text { b. Planned comparisons between intellectually matched participants with schizophrenia and controls indicated that there were more males than females in the stable poor group } \\
\text { with schizophrenia compared with the stable poor controls (Fisher's exact } P=0.04 \text { ). } \\
\text { c. Planned comparisons between intellectually matched participants with schizophrenia and controls showed no statistically significant differences or trends in ethnicity (all } P>0.10) \text { ). } \\
\text { d. Planned comparisons between intellectually matched participants with schizophrenia and controls showed no statistically significant differences or trends in years of education } \\
\text { or highest level of education attained (all } P>0.10) \text {. } \\
\text { e. Planned post hoc contrasts between intellectually matched participants with schizophrenia and controls showed no statistically significant differences or trends in NART-R IQ } \\
\text { (all } P>0.0 .10) \text {. } \\
\text { f. Planned post hoc contrasts between intellectually matched participants with schizophrenia and controls showed no statistically significant differences or trends in WAIS-R IQ } \\
\text { (all } P>0.10) \text {. } \\
\text { g. Planned post hoc contrasts between intellectually matched participants with schizophrenia and controls showed no statistically significant differences or trends in NART-R IQ } \\
\text { minus WAIS-R IQ (all } P>0.10) \text {. }\end{array}$} \\
\hline
\end{tabular}




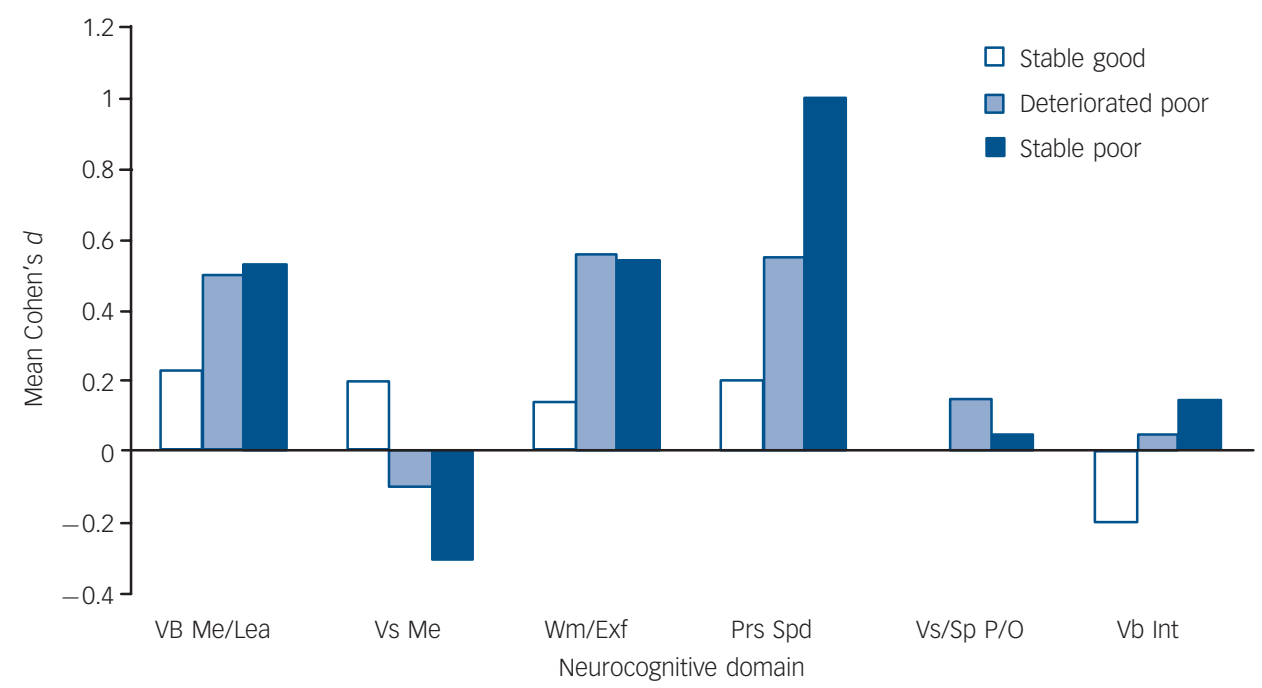

Fig. 1 Mean Cohen's $d$ per neurocognitive domain in the IQ subgroups for participants with schizophrenia.

Vb Me/Lea, Verbal memory and learning; Vs Me, Visual memory; Wm/Exf, Working memory and executive function; Prs Spd, Processing speed; Vs/Sp P/O, Visuospatial perception and organisation; $\mathrm{Vb}$ Int, Verbal intelligence.

and controls. A visual display of the average effect sizes across all component processes in each neuropsychological domain in the three groups with schizophrenia is presented in Fig. 1.

The ANCOVAs revealed significant differences across groups in all the neuropsychological variables (Table 2). Compared with intellectually matched controls, all groups with schizophrenia showed significant $(P \leqslant 0.05-0.001)$ or suggestive $(P<0.10)$ deficits, and/or moderate to large effect sizes in verbal memory and learning, working memory, executive function, and processingspeed variables (Table 2). The mean effect size across the variables that showed suggestive or significant deficits, and/or moderate to large effect sizes (Table 2), ranged from 0.4 (small) in the stable good group, to 0.7 (medium) in the deteriorated poor group, to 0.9 (large) in the stable poor group.

\section{Role of working memory and processing speed}

It has been argued that 'core' deficits in working memory and processing speed ${ }^{4,28-30}$ may underlie impairments in several other cognitive domains in schizophrenia. To test this hypothesis, the ANCOVAS and post hoc contrasts between intellectually matched cases and controls were repeated for those neurocognitive variables that gave rise to significant/suggestive deficits and/or medium/large effect sizes (Table 2) while covarying separately for working memory (Letter-number span) (Table 3) and processing speed (Trail Making - part A and Digit symbol) (Table 4). Addition of either covariate to the analysis only slightly modified the pattern of findings (effect sizes in verbal memory and learning decreased slightly) (Tables 3 and 4). The same pattern was observed after controlling for Category and Letter fluency, the executive components that elicited the largest deficit in the 'poor' groups (results available on request).

\section{Role of disease-related variables}

No significant associations emerged between cognitive impairment and medication dose, positive symptoms or depressive symptoms in the combined sample of individuals with schizophrenia (stable good, deteriorated poor, stable poor combined). Higher levels of negative symptoms correlated with poorer verbal learning (Pearson $r=-0.50, P=0.0001$ ), short-term verbal recall (Pearson $r=-0.29, P=0.04$ ), delayed verbal recall (Pearson $r=-0.29, P=0.04$ ) and working memory (Pearson $r=-0.31, P=0.02)$. Longer duration of untreated psychosis was associated with poorer short-term (Pearson $r=-0.30, P=0.02$ ) and delayed (Pearson $r=-0.28, P=0.04$ ) verbal recall.

To examine whether negative symptoms could account for the deficits in verbal learning, verbal recall and working memory in the groups with schizophrenia, we compared these domains between individuals with subclinical negative symptoms (18 stable good, 24 deteriorated poor, 10 stable poor) and intellectually matched controls. The pattern of findings as outlined in Table 2 remained the same (results available on request).

\section{Examining potential biases in intellectual classification}

The formation of intellectual subgroups in the present study was based on a priori defined, previously validated, criteria for a meaningful discrepancy between premorbid and current IQ. ${ }^{2-5,16,21}$ However, the percentage of controls showing a decline of at least 10 IQ points from premorbid levels (22\%) was higher than expected. ${ }^{16}$ We therefore repeated the classification and analysis using a considerably more conservative cut-off for premorbid/current IQ discrepancy (17 points), as less than $10 \%$ of our controls showed a NART-R minus WAIS-R discrepancy of at least this magnitude. The pattern of findings as outlined in Table 2 remained the same (results available on request).

To address the possibility that current IQ was overestimated in the individuals with schizophrenia (WAIS-R Vocabulary is considered to be relatively resistant to brain damage), ${ }^{31}$ participants were reclassified to intellectual subgroups using an estimate of WAIS-R IQ based on Comprehension and Digit symbol (the most sensitive subtest to brain damage). ${ }^{31}$ This led to an average decrease of 3.0 points in the current IQ of the participants with schizophrenia, but, again, did not affect the pattern of findings (results available on request).

\section{Using continuous IQ predictors of neuropsychological function}

As mentioned earlier, $8 \%$ of the 101 people with schizophrenia and $14 \%$ of the 317 controls did not fall in any a priori defined 


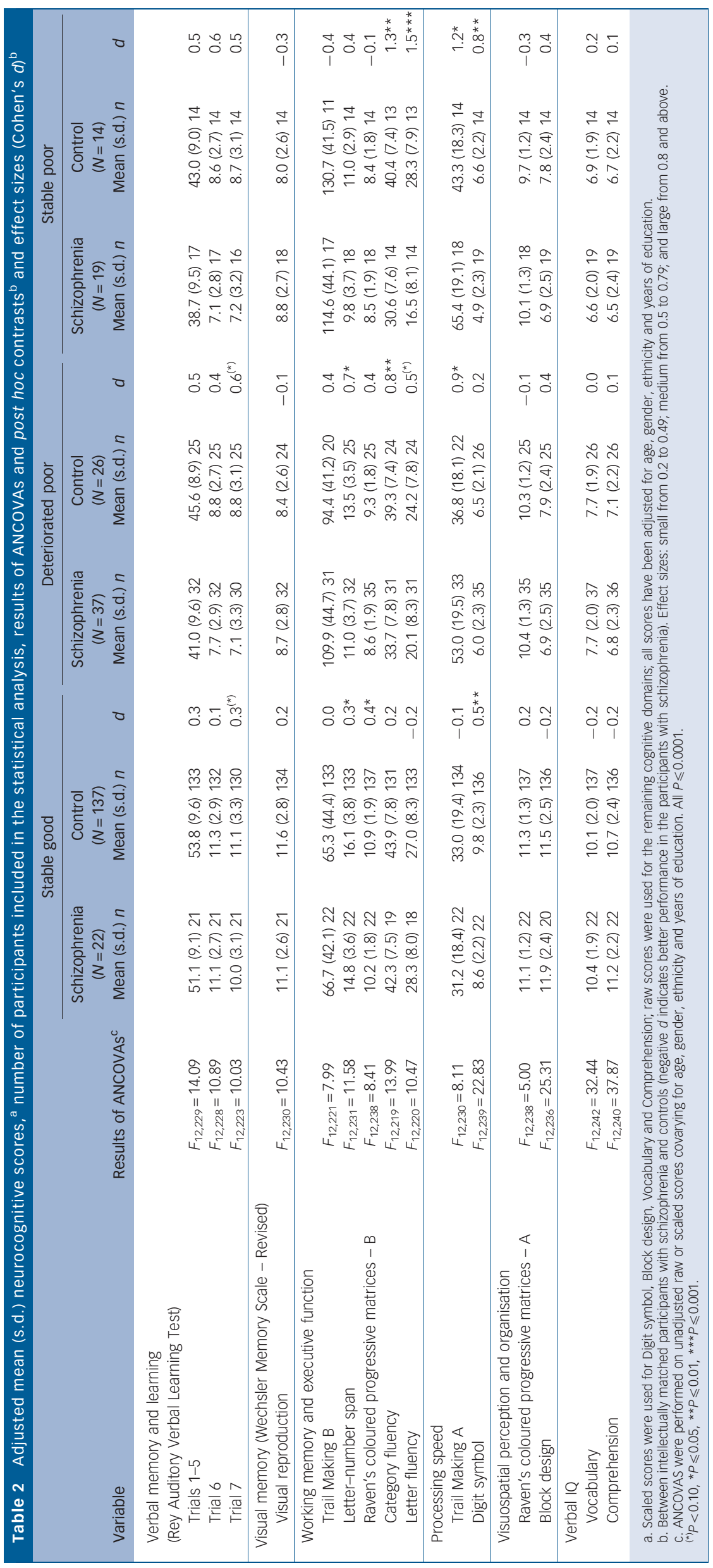



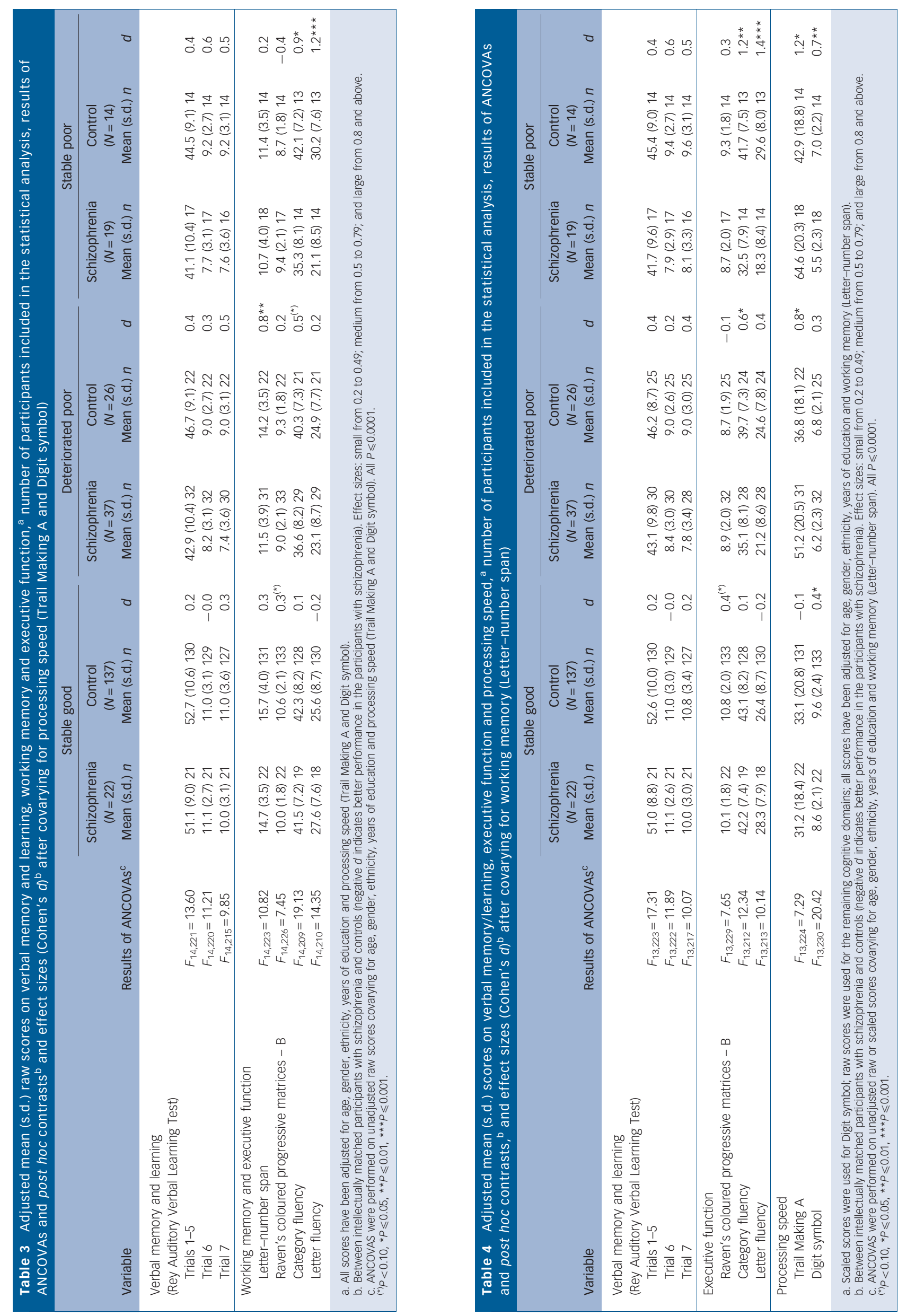
IQ category, and $15 \%$ of the individuals with schizophrenia (and their intellectually matched controls) were excluded from the analysis because of the small sizes of their representative intellectual categories. To address this methodological downside, we carried out an alternative analysis: the effects of WAIS-R IQ and premorbid/current IQ discrepancy (NART-R IQ minus WAIS-R IQ) on neurocognitive function were simultaneously addressed in multiple regression models in each of the total schizophrenia $(n=101)$ and control $(n=317)$ samples, covarying for age, gender, ethnicity and years of education. The independent effect of WAIS-R IQ was statistically significant for all neurocognitive variables ( $\alpha$ was set at $0.05, P<0.05-0.001$ ), contributing $6-39 \%$ of unique variance in the participants with schizophrenia, and $2-22 \%$ of unique variance in the controls. The independent effect of NART-R IQ minus WAIS-R IQ was statistically significant for a smaller set of functions $(P<0.05-0.01)$, contributing $0-10 \%$ of unique variance in the participants with schizophrenia, and $0-4 \%$ of unique variance in the controls (results available on request). These findings suggest that current IQ and IQ trajectory may have independent roles in neuropsychological function. They further suggest a relatively greater contribution of current IQ compared with IQ trajectory, and a somewhat greater explanatory power in relation to the performance of the participants with schizophrenia than that of the controls' performance.

\section{Discussion}

\section{Main findings}

This is the first population-based study to investigate neuropsychological function in people with first-episode schizophrenia and control subgroups that mirrored each other in both intellectual trajectory and current IQ. The epidemiological framework, large and well-characterised sample, novel matching strategy and use of standard cognitive tests in an original and informative way, give our study a powerful foundation and fills a gap in the literature, while supporting previous findings. Our strategy enabled us to isolate neuropsychological deficits in the cohorts of individuals with schizophrenia that were unlikely to have simply resulted from a generic effect of low/lowered IQ on neurocognitive function, sampling biases, long-standing clinical illness, medication or state-dependent factors. We were further able to investigate the utility of intellectual factors in defining meaningful schizophrenia subgroups, whose IQ trajectory and neuropsychological function can inform current theoretical accounts of the developmental and pathophysiological heterogeneity of schizophrenia.

A number of tentative conclusions can be drawn from the present findings. First, teasing out the effects of different aspects of intellectual performance, our study corroborated findings from earlier studies of schizophrenia, which variably identified 'core' deficits in executive function, ${ }^{3-6}$ processing speed $^{4,32}$ or verbal declarative memory. ${ }^{6,33,34}$ Combining statistical tests and effect sizes, our approach demonstrated that deficits in all the above domains are likely to be prototypical of schizophrenia. Second, impairments in the respective domains may reflect independent pathophysiological processes. Although deficits in working memory and processing speed have been suggested to cause cascading effects on other neurocognitive functions, ${ }^{29,32,35,36}$ controlling for either process or for verbal fluency (the most impaired executive component in the 'poor' groups) did not abolish deficits in the remaining domains. Third, visual memory, visuospatial organisation and verbal intellectual ability are relatively spared in schizophrenia, as the respective effect sizes were small (or even negative) and non-significant across the schizophrenia groups. Finally, the graded pattern of average effect sizes across the affected domains in the stable good, deteriorated poor and stable poor groups (ranging from a small 0.4 to a medium 0.7 to a large 0.9 ) suggests that the 'normal' relationship between IQ and neuropsychological function breaks down incrementally in schizophrenia as one moves from high to low current and premorbid IQ.

Our analysis suggested that current IQ and IQ trajectory may have independent roles in neuropsychological function. This result parallels a finding by Kremen and colleagues, ${ }^{37}$ who reported that a static intellectual deficit at age 7, and a large IQ decline between ages 4 and 7 against a background of normal IQ, were both associated with adult risk for psychosis. The above evidence suggests that IQ tests are indexing something fundamental about schizophrenia, and that the neurodevelopmental substrates of the disorder involve both static and dynamic elements, which may dissociate with different subtypes of the disorder. This issue is further discussed below.

\section{Intellectual and neurocognitive subtypes in schizophrenia}

Our findings corroborate earlier attempts at subtyping schizophrenia using intellectual criteria. ${ }^{3-6}$ In particular, the proportions of individuals falling in the stable good, deteriorated good/poor (collapsed in previous studies) and stable poor categories are comparable with those reported in earlier investigations. ${ }^{3-6}$

About a quarter of people with schizophrenia are reported to obtain normal scores on global measures of intellectual or neuropsychological functioning. ${ }^{3,9,10}$ This sizeable minority ( $22 \%$ in our study) showed small isolated deficits in verbal memory, working memory and executive function and a moderate deficit in processing speed. These findings lend support to the argument that the corresponding group might be more accurately referred to as 'high-functioning' rather than neuropsychologically intact, as these individuals show deficits in a restricted range of cognitive functions in several studies. ${ }^{9}$

A substantial proportion of participants with schizophrenia (37\%) showed below average intellectual functioning and a meaningful estimated decline from premorbid levels. Their neuropsychological impairment encompassed the same broad neurocognitive domains as those showing deficits in the stable good group, but involved more subcomponent processes and larger effect sizes. Our findings concur with those of a population-based study in Israel, which demonstrated that $40 \%$ of 17-year-olds with a later admission to hospital for schizophrenia had experienced IQ decline from childhood to adolescence. ${ }^{8}$ It is tempting to view this group as representing a 'late neurodevelopmental' subtype of schizophrenia. ${ }^{8}$ The latter has been hypothesised to involve a genetic regulatory defect in brain development, exerting active effects in adolescence and early adulthood. ${ }^{37,38}$ The plausibility of this form is supported by evidence suggesting that some brain maturational events of adolescence (e.g. electrophysiological changes) are abnormal in about $40 \%$ of people with schizophrenia; ${ }^{38,39}$ and the distinctiveness of this form is supported by findings that individuals with deteriorating intellectual functions have more chronic symptoms and are less responsive to treatment. ${ }^{40,41}$ Importantly, both our study and the Israeli series suggest that the late neurodevelopmental subtype might be the predominant form of schizophrenia.

A sizeable minority of the cohort of individuals with schizophrenia (19\%) showed below-average IQ and a stable IQ trajectory. These people demonstrated deficits in the same broad neurocognitive domains as those eliciting impairments in the stable good and deteriorated poor groups. Their deficit, however, involved more subcomponent functions and larger effect sizes 
compared with the former groups. These individuals may be the prototypical 'early neurodevelopmental' group, in which a deviation in early development as a result of genetic and/or environmental factors establishes a neuronal phenotype (static encephalopathy) that predisposes to, or determines, the onset of schizophrenia. ${ }^{42-44}$ According to this model, the period during which the causative agent actively damages the central nervous system is short (the pathogenetic process is virtually static) and any behavioural consequences remain relatively latent until the onset of psychosis.

In the participants with schizophrenia in this study the likelihood of falling in the stable poor category was about half that of falling in the deteriorated poor group. In line with this finding, Kremen and colleagues reported that the rate of future psychosis among 7-year-olds with low but stable IQ was about half that among peers with normal but deteriorated IQ. ${ }^{37}$ Both studies suggest that a declining IQ trajectory during development is a stronger risk factor for adult schizophrenia than a static intellectual deficiency.

\section{Using the NART-R and the WAIS-R to measure intellectual change}

The NART-R has been validated against the WAIS-R, ${ }^{16}$ and, in healthy control samples, NART-R IQs and WAIS-R IQs differ by an average of $1.0-3.8$ points. ${ }^{5,45,48}$ This range is comparable to the NART-R minus WAIS-R difference of 3.4 points found in our extended control sample. However, several drawbacks need to be acknowledged. In particular, the NART-R should be used with caution where IQ deviates from general population means, as it may over- or underestimate premorbid IQ. ${ }^{46,47}$ Importantly, the latter possibility is more salient in people with first-episode schizophrenia compared with controls, as education is likely to be interrupted and adult vocabulary incompletely attained in the former group. In contrast to earlier studies, which compared IQ subgroups of participants with schizophrenia with a single, intellectually normal, healthy control group, the present study compared subgroups of people with schizophrenia and controls that did not differ in current IQ, level of education or vocabulary.

The percentage of controls showing cognitive instability in our study was relatively high: about twice as many ÆSOP controls (22\%) showed a 'deficit' of at least 10 points relative to their predicted IQ as participants in the NART restandardisation study $(10 \%) .{ }^{16}$ Differences in the characteristics of the NART-R sample and the ÆSOP controls may partly explain this finding. In particular, individuals with high IQ were over-represented in the NART-R sample compared with the general population, ${ }^{16}$ whereas $Æ S O P$ controls were epidemiologically ascertained. The presence of non-psychotic psychiatric disorders in the ÆSOP controls may have increased rates of cognitive decline compared with the NART-R sample. However, an alternative explanation is that score differences between the NART-R and the WAIS-R reflect differences between verbal and non-verbal, or acquired and innate, cognitive abilities, or simply measurement error. In particular, the NART-R measures only acquired verbal function, whereas the WAIS-R measures both verbal and non-verbal IQ, and aspects that are more and less acquired. Given the different psychometric properties of the two instruments, and the lack of prospective studies which support their combined use in the study of intellectual change, the validity and reliability of this approach remain to be established.

\section{Other methodological considerations}

Comparing symptoms, disease course, genetic indices or brain anatomical and functional measures between intellectual subtypes was beyond the scope of the present investigation. However, such strategy would have enhanced the interpretability of our findings in relation to the neurobiological or nosological 'signature' of the subtypes investigated. For example, Turetsky et $\mathrm{al}^{11}$ have reported 'memory' subtypes of schizophrenia to have distinct clinical, neuroanatomical and neurophysiological correlates. Albeit not cross-referenced with neurobiological investigations, the meaningfulness of subtyping individuals with schizophrenia based on IQ level and trajectory was supported by our neuropsychological findings, as well as results of regression analysis of continuous intellectual predictors. The latter approach further enabled us to address the 'loss' of some participants during subtyping.

To increase the distinctiveness and face validity of the IQ categories, the present study used more refined criteria for intellectual classification than those used in previous studies. ${ }^{3-5}$ The 'deteriorated good' participants were not lumped together with the 'deteriorated poor' individuals as in previous studies, ${ }^{3,4}$ since the differential ratio of deteriorated good/poor participants in the schizophrenia (0.2) and control (1.7) samples would have confounded the statistical comparison.

Performance on the WAIS-R was used to define both our matching criteria (full-scale IQ and IQ decline) and four outcome variables (Digit symbol, Block design, Vocabulary and Comprehension). As matching for WAIS-R IQ does not ensure equivalence on the cognitive components of general ability, ${ }^{49}$ examining the neurocognitive processes measured by the four subtests was central to our discussion of relative deficits in schizophrenia. The findings of the present study closely parallel those of a recent investigation. ${ }^{49}$ Leeson and colleagues compared individuals with first-episode psychosis and healthy controls, one-to-one matched for full-scale current IQ, on WAIS subtests of perceptual organisation, verbal comprehension, processing speed and working memory, as well as other tests of executive function and episodic memory. ${ }^{49}$ In line with the present findings, the groups showed equivalent performance on all WAIS subtests except Digit symbol processing speed, on which the individuals with schizophrenia performed significantly worse. ${ }^{49}$ In addition, covarying for processing speed did not abolish the deficit in working memory and verbal learning of the participants with schizophrenia. ${ }^{49}$

Although the deficit in processing speed of the participants with schizophrenia was a robust finding, and our analysis corrected for age, we cannot exclude the possibility that the age 'advantage' of the stable good and the deteriorated poor schizophrenia subgroups relative to intellectually matched controls moderated the effect detected for processing speed, which is age-dependent (both the stable good and the deteriorated poor schizophrenia subgroups showed deficits in one test of processing speed, as opposed to the stable poor schizophrenia subgroup, who showed deficits in two tests).

Identifying 'specific' cognitive deficits in schizophrenia is a long-standing and controversial issue, which requires the use of theoretically relevant measures that do not 'just correlate with IQ'. Adding to the complexity of the problem is the attempt to measure the same neurocognitive domain using more than one test, as this requires psychometrically matched instruments with comparable discriminating power. Failing to satisfy this criterion (or natural variations across samples) may explain why the same domain elicits deficits in some tests but not others. The use of psychometric tests with different reliabilities and discriminating powers poses a particular problem in studies that attempt to identify specific cognitive deficits in a disorder by trying to establish whether participants with schizophrenia and controls differ more on some theoretically relevant measures (focal tasks) than on control tasks measuring other constructs (reference tasks). 
Alternative methods proposed for overcoming this problem include matching participants with schizophrenia and controls on a highly reliable variable such as current IQ (averting confounds resulting from general performance deficiencies in schizophrenia), analysis of covariance (do statistical differences on the focal task remain after controlling for performance on control tasks?), process-oriented solutions (using well-established models from cognitive psychology to predict specific theorydriven patterns of performance within and across tasks) and psychometric matching. The current study adopted both IQmatching and analysis of covariance. However, both methods have been criticised as much as process-oriented solutions and psychometric matching, and, currently, there is no unequivocally accepted method for establishing specific cognitive deficits in a disorder. For a further discussion of this important topic, the reader is referred to a special section of the Journal of Abnormal Psychology. ${ }^{50-54}$

The interpretation of effect sizes generally depends on the assumptions that the schizophrenia group and control group values are normally distributed and have the same standard deviations. Both assumptions were tenable for the majority, but not all, of our tasks, reducing the comparability of deficits across neuropsychological components.

In summary, subtyping schizophrenia on the basis of current IQ and IQ trajectory offers a potential means for resolving the phenotypic complexity of the disorder, and is in line with current theoretical models of developmental heterogeneity in schizophrenia. Our findings are in agreement with those of previous studies $^{3-5,9,13}$ in suggesting that IQ decline in schizophrenia is not universal. Deficits in executive function, processing speed and verbal memory appear to be prototypical of the disorder, and may coexist with preserved IQ. Our findings further suggest that these key deficits may reflect independent pathophysiological processes.

Eugenia Kravariti, MA, MSC, PhD, Department of Psychiatry, NIHR Biomedical Research Centre for Mental Health, South London and Maudsley NHS Foundation Trust and Institute of Psychiatry, King's College London, London; Kevin Morgan PhD, Department of Psychiatry, NIHR Biomedical Research Centre for Mental Health South London and Maudsley NHS Foundation Trust and Institute of Psychiatry, King's College London and Department of Psychology, University of Westminster London; Paul Fearon, MSC, PhD, MRCPI, MRCPsych, Jolanta W. Zanelli, MSC, Julia M. Lappin, MBChB, MSc, MRCPsych, Paola Dazzan, MSC, PhD, MRCPsych, Craig
La Morgan, MSC, PhD, Department of Psychiatry, NIHR Biomedical Research Centre for Mental Health, South London and Maudsley NHS Foundation Trust and Institute of Psychiatry, King's College London, London; Gillian A. Doody, MPhil, MRCPsych, Community Health Sciences, Queen's Medical Centre, Institute of Clinical Research, University of Nottingham, Nottingham; Glynn Harrison, FRCPsych, Academic Unit of Psychiatry, University of Bristol, Bristol; Peter B. Jones, MSC, PhD, FRCP, MRCPsych, Department of Psychiatry, University of Cambridge, Cambridge; Robin M. Murray, Department of Psychiatry, University of Cambridge, Cambridge; Robin M. Murray
DSC, FRCP, FRCPsych, FmedSci, Abraham Reichenberg, PhD, Department of Psychiatry, NIHR Biomedical Research Centre for Mental Health, South London and Maudsley NHS Foundation Trust and Institute of Psychiatry, King's College London, London, UK

Correspondence: Eugenia Kravariti, PhD, Department of Psychiatry, Box 58 NIHR Biomedical Research Centre for Mental Health, South London and Maudsley NHS Foundation Trust and Institute of Psychiatry, King's College London, De Crespigny Park, London SE5 8AF, UK. Email: e.kravariti@iop.kcl.ac.uk

First received 1 Jun 2008, final revision 3 Apr 2009, accepted 6 May 2009

\section{Funding}

The study was funded by grants from the Medical Research Council, London, England, and the Stanley Medical Research Institute, Bethesda, Maryland, USA. Both funding organisations provided financial support for the conduct of study, collection, management and analysis of data.

\section{Acknowledgements}

We would like to thank the staff in the mental health services who helped in the case ascertainment and the research participants. We gratefully acknowledge advice from the late R. E. Kendell, FRCPsych, regarding the design of the study. We wish to acknowledge the contributions of the entire $\mathbb{E S O P}$ study team, listed online at http://www.psychiatry. cam.ac.uk/aesop.

\section{References}

1 Heinrichs RW. In Search of Madness. Oxford University Press, 2001.

2 Seidman LJ, Buka SL, Goldstein JM, Tsuang MT. Intellectual decline in schizophrenia: evidence from a prospective birth cohort 28 year follow-up study. J Clin Exp Neuropsychol 2006; 28: 225-42.

3 Weickert TW, Goldberg TE, Gold JM, Bigelow LB, Egan MF, Weinberger DR. Cognitive impairments in patients with schizophrenia displaying preserved and compromised intellect. Arch Gen Psychiatry 2000; 57: 907-13.

4 Badcock JC, Dragovic M, Waters FAV, Jablensky A. Dimensions of intelligence in schizophrenia: evidence from patients with preserved, deteriorated and compromised intellect. J Psychiatr Res 2005; 39: 11-9.

5 Joyce EM, Hutton SB, Mutsatsa SH, Barnes TRE. Cognitive heterogeneity in first-episode schizophrenia. Br J Psychiatry 2005; 187: 516-22.

6 Kremen WS, Seidman LJ, Faraone SV, Tsuang MT. Intelligence quotient and neuropsychological profiles in patients with schizophrenia and in normal volunteers. Biol Psychiatry 2001; 50: 453-62.

7 Kremen WS, Seidman LJ, Faraone SV, Tsuang MT. IQ decline in crosssectional studies of schizophrenia: methodology and interpretation. Psychiatry Res 2008; 158: 181-94.

8 Reichenberg A, Weiser M, Rapp MA, Rabinowitz J, Caspi A, Schmeidler J, et al. Elaboration on premorbid intellectual performance in schizophrenia: premorbid intellectual decline and risk for schizophrenia. Arch Gen Psychiatry 2005; 62: 1297-304.

9 Allen DN, Goldstein G, Warnick E. A consideration of neuropsychologically normal schizophrenia. J Int Neuropsychol Soc 2003; 9: 56-63.

10 Palmer DW, Heaton RK, Paulsen JS, Kuck J, Braff D, Harris MJ, et al. Is it possible to be schizophrenic yet neuropsychologically normal? Neuropsychology 1997; 11: 437-46.

11 Turetsky BI, Moberg PJ, Mozley LH, Moelter ST, Agrin RN, Gur RC, et al. Memory-delineated subtypes of schizophrenia: relationship to clinical, neuroanatomical, and neurophysiological measures. Neuropsychology 2002; 16: $481-90$.

12 Rabinowitz J, De Smedt G, Harvey PD, Davidson M. Relationship between premorbid functioning and symptom severity as assessed at first episode of psychosis. Am J Psychiatry 2002; 159: 2021-6.

13 van Winkel R, Myin-Germeys I, Delespaul P, Peuskens J, De HM, van OJ. Premorbid IQ as a predictor for the course of IQ in first onset patients with schizophrenia: a 10-year follow-up study. Schizophr Res 2006; 88: 47-54.

14 World Health Organization. The ICD-10 Classification of Mental and Behavioural Disorders: Clinical Descriptions and Diagnostic Guidelines. WHO, 1992.

15 Morgan C, Dazzan P, Morgan K, Jones P, Harrison G, Leff J, et al. First episode psychosis and ethnicity: initial findings from the AESOP study. World Psychiatry 2006; 5: 40-6.

16 Nelson HE WJ. National Adult Reading Test Manual (2nd edn). nferNelson, 1991.

17 Wechsler D. Manual for the Wechsler Adult Intelligence Scale - Revised. Psychological Corporation, 1981.

18 World Health Organization. SCAN (Schedules for Clinical Assessment in Neuropsychiatry). WHO, 1992.

19 Bebbington PE, Nayani T. The psychosis screening questionnaire. Int J Method Psych 1995; 5: 11-20.

20 Mallett R. Sociodemographic Schedule. Section of Social Psychiatry, Institute of Psychiatry, 1997.

21 Goldberg TE, Torrey EF, Gold JM, Bigelow LB, Ragland RD, Taylor E, et al. Genetic risk of neuropsychological impairment in schizophrenia - a study of monozygotic twins discordant and concordant for the disorder. Schizophr Res 1995; 17: 77-84.

22 Spreen O, E Strauss. A Compendium of Neuropsychological Tests: Administration, Norms and Commentary. Oxford University Press, 1991.

23 Wechsler D. Wechsler Memory Scale - Revised Manual. Psychological Corporation, 1987.

24 Reitan RM. Validity of the Trail Making Test as an indicator of organic brain damage. Percept Mot Skills 1958; 8: 271-6.

25 Gold JM, Carpenter C, Randolph C, Goldberg TE, Weinberger DR. Auditory working memory and Wisconsin card sorting test performance in schizophrenia. Arch Gen Psychiatry 1997; 54: 159-65.

26 Raven JC. Coloured Progressive Matrices. Sets A, AB, B. Oxford Psychologists Press, 1995 
27 Cohen J. A power primer. Psychol Bull 1992; 112: 155-9.

28 Silver H, Feldman P, Bilker W, Gur RC. Working memory deficit as a core neuropsychological dysfunction in schizophrenia. Am J Psychiatry 2003; 160 1809-16.

29 Mitropoulou V, Harvey PD, Zegarelli G, New AS, Silverman JM, Siever L. Neuropsychological performance in schizotypal personality disorder: importance of working memory. Am J Psychiatry 2005; 162: 1896-903.

30 Dickinson D, Ramsey ME, Gold JM. Overlooking the obvious: a meta-analytic comparison of digit symbol coding tasks and other cognitive measures in schizophrenia. Arch Gen Psychiatry 2007; 64: 532-42.

31 Bilder RM, Lipschutzbroch L, Reiter G, Geisler SH, Mayerhoff DI, Lieberman JA. Intellectual deficits in 1st-episode schizophrenia - evidence for progressive deterioration. Schizophr Bull 1992; 18: 437-48.

32 Rodríguez-Sánchez JM, Crespo-Facorro B, González-Blanch C, Perez-Iglesias $R$, Vázquez-Barquero JL. Cognitive dysfunction in first-episode psychosis: the processing speed hypothesis. Br J Psychiatry 2007; 191 (suppl 51): s107-10.

33 Saykin AJ, Gur RC, Gur RE, Mozley D, Mozley LH, Resnick SM, et al. Neuropsychological function in schizophrenia: selective impairment in learning and memory. Arch Gen Psychiatry 1991; 48: 618-24.

34 Hoff AL, Kremen WS. Is there a cognitive phenotype for schizophrenia: the nature and course of the disturbance in cognition? Curr Opin Psychiatr 2002 15: 43-8.

35 Brebion G, David AS, Bressan RA, Pilowsky LS. Role of processing speed and depressed mood on encoding, storage, and retrieval memory functions in patients diagnosed with schizophrenia. J Int Neuropsychol Soc 2007; 13: 99-107.

36 Elvevag B, Goldberg TE. Cognitive impairment in schizophrenia is the core of the disorder. Crit Rev Neurobiol 2000; 14: 1-21.

37 Kremen WS, Buka SL, Seidman L, Goldstein JM, Koren D, Tsuang MT. IQ decline during childhood and adult psychotic symptoms in a community sample: a 19-year longitudinal study. Am J Psychiatry 1998; 155: 672-7.

38 Feinberg I. Schizophrenia: caused by a fault in programmed synaptic elimination during adolescence? J Psychiatr Res 1982; 17: 319-34.

39 Woods BT. Is schizophrenia a progressive neurodevelopmental disorder? Toward a unitary pathogenetic mechanism. Am J Psychiatry 1998; 155: 1661-70.

40 Rabinowitz J, Haim R, Reichenberg A, Harvey PD, Weiser M, Kaplan Z. Premorbid functioning and outcome in first episode schizophrenia. Schizophr Res 2004; 67 (suppl 1): 37.
41 Harvey PD, Silverman JM, Mohs RC, Parrella M, White L, Powchik P, et al. Cognitive decline in late-life schizophrenia: a longitudinal study of geriatric chronically hospitalized patients. Biol Psychiatry 1999; 45: 32-40.

42 Weinberger DL. Implications of normal brain development for the pathogenesis of schizophrenia. Arch Gen Psychiatry 1987; 44: 660-9.

43 Murray RM. Neurodevelopmental schizophrenia: the rediscovery of dementia praecox. Br J Psychiatry 1994; 165 (suppl 25): s6-12.

44 Murray RM, O'Callaghan E, Castle DJ, Lewis SW: A neurodevelopmental approach to the classification of schizophrenia. Schizophr Bull 1992; 18: 319-32.

45 Bright $P$, Jaldow E, Kopelman MD. The National Adult Reading Test as a measure of premorbid intelligence: a comparison with estimates derived from demographic variables. J Int Neuropsychol Soc 2002; 8: 847-54.

46 Russell AJ, Munro J, Jones PB, Hayward P, Hemsley DR, Murray RM. The National Adult Reading Test as a measure of premorbid IQ in schizophrenia. Br J Clin Psychol 2000; 39 (Pt 3): 297-305.

47 Graves RE. Accuracy of regression equation prediction across the range of estimated premorbid IQ. J Clin Exp Neuropsychol 2000; 22: 316-24.

48 Berry DT, Carpenter GS, Campbell DA, Schmitt FA, Helton K, Lipke-Molby T. The New Adult Reading Test-Revised: accuracy in estimating WAIS-R IQ scores obtained 3.5 years earlier from normal older persons. Arch Clin Neuropsychol 1994; 9: 239-50.

49 Leeson VC, Barnes TRE, Harrison M, Matheson E, Harrison I, Mutsatsa SH, et al. The relationship between IQ, memory, executive function, and processing speed in recent-onset psychosis: 1-year stability and clinical outcome. Schizophr Bull 2008; Aug 4 (Epub ahead of print).

50 Strauss ME. Methodology for identifying specific psychological deficits: introduction to the special section. J Abnorm Psychol 2001; 110: 4-5.

51 Strauss ME. Demonstrating specific cognitive deficits: a psychometric perspective. J Abnorm Psychol 2001; 110: 6-14.

52 Knight RA, Silverstein SM. A process-oriented approach for averting confounds resulting from general performance deficiencies in schizophrenia. J Abnorm Psychol 2001; 110: 15-30.

53 Chapman $\sqcup$, Chapman JP. Commentary on two articles concerning generalized and specific cognitive deficits. J Abnorm Psychol 2001; 110: 31-9.

54 Miller GA, Chapman JP. Misunderstanding analysis of covariance. J Abnorm Psychol 2001; 110: 40-8. 